\title{
29 new variable stars near Nova Sagittarii.
}

\section{(Harvard College Observatory Circular No. I4r).}

In the study of the distribution of variable stars now in progress at this Observatory, an important line of research has for its object the discovery of groups of faint variables. At present, the most remarkable regions known are certain globular clusters, the Nebula of Orion, and the Magellanic Clouds. The investigation here referred to does not include the globular clusters. The photographs employed were taken with the 24-inch Bruce Telescope and have long exposures, preferably two hours, or more. The choice of the particular region to be examined at any time is guided principally by the number and quality of photographs available for com. parison, preference being given to regions which are in themselves especially interesting. No attempt has been made to follow any consecutive order.

The region near Nova Sagittarii has recently been examined, by Miss Leavitt, on three Bruce plates having their centres for 1855 , in RA. $=18^{\mathrm{h}} 49^{\mathrm{m}}$, Decl. $=-12{ }^{\circ}$; RA. $=18^{\mathrm{h}} 54^{\mathrm{m}}$, Decl. $=-122_{2} ;$ RA. $=18^{\mathrm{h}} 55^{\mathrm{m}}$, Decl $=-13^{\circ} 4$. The corresponding plates, dates, and exposures are A 2845, Oct. $23,1897,60^{\mathrm{m}}$; $\mathrm{A}_{4091}$, Oct. 26 , 1899 , I $50^{\mathrm{m}}$; A 5712 , Oct. 10, r $90 \mathrm{r}, 180^{\mathrm{m}}$. No two of the plates have the same centre. The region common to the three extends in right ascension, from $18^{\mathrm{h}} 42^{\mathrm{m}}$ to $19^{\mathrm{h}} 2^{\mathrm{m}}$, and in declination from - $10 \% 9$ to $-14 \% 7$, while the extreme limits of the region which may be compared on any two plates are $18^{\mathrm{h}} 41^{\mathrm{m}}$ and $19^{\mathrm{h}} 7^{\mathrm{m}}$ in right ascension, and -10 :0 and $-15 \%$ in declination. As there is much difficulty in comparing star images on superposed Bruce plates when the centres are a degree or more apart, it is not probable that all stars showing variation on these plates were discovered. Nevertheless, a surprising number of faint variables was found. The material existing for their confirmation is scanty, consisting principally of the three Bruce plates above mentioned, and three plates taken with the 8-inch Bache Telescope. The designations, dates, and exposures of these plates are, B 23759 , Aug. 2, $1899,153^{\mathrm{m}}$; B 32278 , July $17,1903,60^{\mathrm{m}}$; B 32341 , July 28, r $903,60^{\mathrm{m}}$. Seven other Bache plates were examined for a few of the brightest variables. Nova Sagittarii and the known variable, ST Sagittarii, were rediscovered. The stars which show well-defined changes, and are certainly bright on at least two plates, and certainly faint on at least two, are given in Table $I$. The first five columns give the designation, the Harvard number, the provisional designation in $A$. N., the right ascension for 1900 , and the declination for 1900 . The sixth to the eleventh columns give the observations on the six plates described above. The range is given in the last column. As a rule, only two estimates of the actual magnitudes were made, but each variable was looked for on each plate and recorded as bright or faint. The reason for printing these rough observations is the probability that it may be a considerable time before a sufficient number of photographs can be obtained for the detailed study of the variables.

On Plate B 30495 , taken July 30,1902 , exposure $60^{\mathrm{m}}$, the magnitude of 78.1908 Sagittarii was estimated as 14.5 .

Table I. New variables.

\begin{tabular}{|c|c|c|c|c|c|c|c|c|c|c|c|}
\hline Design. & H. V. & $\begin{array}{l}\text { Prov. Designation } \\
\text { in A. N. }\end{array}$ & RA. I900 & Decl. I 900 & A 2845 & B 23759 & $A_{4091}$ & A 5712 & B 32278 & B 3234I & Range \\
\hline I 844 I 4n & 3102 & 60.1908 Scuti & $18^{\mathrm{h}} 44^{\mathrm{m}} 3^{8^{\mathrm{s}}}$ & $-14^{\circ} 16^{\prime} 2$ & $<I_{5} .8$ & Ft. & 15.4 & $<16.2$ & Ft. & - & $>0.8$ \\
\hline $184510_{n}$ & 3103 & 61.1908 Scuti & $1845 \quad 2$ & -1050.1 & 14.0 & $\mathrm{Br}$. & Br. & 15.0 & Br. & Ft. & I.0 \\
\hline 1845 I $3 n$ & 3104 & 62.1908 Scuti & $1845 \quad 56$ & -132.6 & 13.2 & 14.0 & - & 10.0 & $\mathrm{Br}$. & Br. & 4.0 \\
\hline 1847 I $2 a_{n}$ & 3105 & 63.1908 Scuti & $1847 \quad 16$ & $\begin{array}{ll}-12 & 37.9\end{array}$ & 13.4 & Ft. & $\mathrm{Br}$. & 15.0 & Br. & Ft. & 1.6 \\
\hline 1847 i $2 b_{n}$ & 3106 & 64.1908 Scuti & $\begin{array}{lll}18 \quad 47 \quad 35\end{array}$ & -1253.7 & Ft. & Ft. & 15.2 & $<16.0$ & Br. & Br. & $>0.8$ \\
\hline 1848 I $4 n$ & 3107 & 65.1908 Scuti & $\begin{array}{lll}18 & 48 & \text { I }\end{array}$ & - I 4 rg.I & $15 \cdot 4$ & - & I 2.0 & 14.0 & Ft. & - & 3.4 \\
\hline 1848 I $2 b_{n}$ & 3108 & 66. I 908 Scuti & $\begin{array}{lll}18 & 48 & 15\end{array}$ & -1238.5 & 15.0 & $\mathrm{Br}$. & $<16.0$ & 15.8 & $\mathrm{Br}$. & $\mathrm{Br}$. & $>1.0$ \\
\hline $184812 c_{n}$ & 3109 & 67.1908 Scuti & $1848 \quad 16$ & -1246.3 & Ft. & I 2.0 & 15.2 & $<16.0$ & Br. & Ft. & $>4.0$ \\
\hline 1849 I In & 3110 & 68. I 908 Scuti & $\begin{array}{lll}18 & 49 & 14\end{array}$ & $\begin{array}{ll}\text { I I } 5.8\end{array}$ & 13.6 & Ft. & Ft. & I 5.5 & $\mathrm{Br}$. & - & 1.9 \\
\hline 1849 r 2n & $31 \mathrm{II}$ & 69.1908 Scuti & $\begin{array}{lll}18 & 49 & 15\end{array}$ & - I 218.8 & I 5.4 & Br. & Ft. & 14.4 & Ft. & Ft. & 1.0 \\
\hline $18511 O_{n}$ & $3 \times 12$ & 70.1908 Scuti & $\begin{array}{lll}18 & 51 & 39\end{array}$ & $-10 \quad 46.2$ & 13.7 & $\mathrm{Br}$. & - & 14.6 & Ft. & Ft. & 0.9 \\
\hline $185212 n$ & $3 \times 3$ & 71.1908 Scuti & $18 \quad 5226$ & - I 235.4 & 15.8 & Ft. & $<16.0$ & I 4.7 & $\mathrm{Br}$. & - & $>\mathbf{I} \cdot \mathbf{3}$ \\
\hline $185315 n$ & $31 \times 4$ & 72.1908 Scuti & 185320 & -158.7 & 15.2 & $\mathrm{Br}$. & - & 14.0 & Ft. & $\mathrm{Br}$. & $\mathbf{r . 2}$ \\
\hline I 853 I 3n & 3115 & 73.1908 Sagittarii & $18533^{6}$ & -1326.7 & Ft. & Ft. & 13.9 & $<15.8$ & Br. & 一 & $>1.9$ \\
\hline $185312 n$ & 3116 & 74.1908 Sagittarii & I8 $53 \quad 57$ & $\begin{array}{ll}-12 & 6.8\end{array}$ & $<\mathrm{I}_{5} .8$ & Ft. & 15.0 & 15.7 & Br. & 14.5 & $>1.3$ \\
\hline $18541 I_{n}$ & 3117 & 75.1908 Aquilae & 185452 & - I 137.7 & 15.8 & 14.8 & Ft. & 15.0 & Ft. & - & 1.0 \\
\hline $185511 n$ & 3118 & 76.1908 Aquilae & 185530 & $\begin{array}{ll}-11 & 3.5\end{array}$ & 13.5 & Ft. & Br. & 14.5 & Ft. & Ft. & 1.0 \\
\hline $185512 b_{n}$ & 3519 & 77.1908 Sagittarii & 185538 & - I 238.3 & Ft. & Ft. & 15.0 & 15.8 & Br. & $\mathrm{Br}$. & 0.8 \\
\hline 1856 I $2 n$ & 3120 & 78.1 908 Sagittarii & $18 \quad 5^{6} 3^{\circ}$ & -1236.8 & Br. & - & $<\mathbf{1 6 . 0}$ & 15.0 & Ft. & Ft. & $>1.5$ \\
\hline $185712 n$ & 3121 & 79.1908 Sagittarii & $18 \quad 5733$ & -1216.0 & Ft. & Ft. & I 4.4 & $<15.8$ & $\mathrm{Br}$. & $\mathrm{Br}$. & $>1.4$ \\
\hline $185814 n$ & 3122 & 80.1908 Sagittarii & 185816 & $\begin{array}{ll}-14 & 0.9\end{array}$ & 15.2 & Ft. & Br. & I 2.I & - & Ft. & 3.I \\
\hline $1858 \times 3 n$ & 3123 & 81.1908 Sagittarii & $\begin{array}{lll}18 & 58 & 35\end{array}$ & $-\mathrm{r} 3 \quad \mathrm{r.6}$ & $\mathrm{Br}$ & $\mathrm{Br}$. & 13.4 & 14.2 & Ft. & Ft. & 0.8 \\
\hline
\end{tabular}




\begin{tabular}{|c|c|c|c|c|c|c|c|c|c|c|c|}
\hline Design. & H. V. & $\begin{array}{l}\text { Prov. Designation } \\
\text { in A.N. }\end{array}$ & RA. I900 & Decl. I900 & A 2845 & В 23759 & A 4091 & A 5712 & B 32278 & B 3234I & Range \\
\hline 1859 I $2 n$ & 3124 & 82.1908 Sagittarii & $18^{\mathrm{b}} 59^{\mathrm{m}} 56^{\mathrm{s}}$ & $-12^{\circ} 50: 8$ & $< \pm 5.8$ & Br. & 16.0 & I 2.0 & Ft. & Ft. & 4.0 \\
\hline $190112 a_{n}$ & 3125 & 83.r 908 Sagittarii & $19 \circ 5^{8}$ & -1225.7 & $\mathbf{F t}$. & $\mathrm{Br}$. & $<\mathbf{r 6 . 0}$ & 15.2 & Ft. & Ft. & $>0.8$ \\
\hline I 90 I $12 b_{n}$ & 3126 & 84.1908 Sagittarii & I 4 & $-12 \quad 29.7$ & $<$ I 5.9 & Ft. & I 5.2 & I 5.6 & $\mathrm{Br}$. & - & $>0.7$ \\
\hline I $90112 C_{n}$ & 3127 & 85.1908 Sagittarii & I I 4 & $\begin{array}{ll}-12 & 3.9\end{array}$ & Ft. & Ft. & 15.0 & I 5.8 & I 5.0 & $\mathrm{Br}$. & 0.8 \\
\hline $190312 n$ & 3128 & 86. I 908 Sagittarii & 323 & $\begin{array}{ll}-12 & 30.7\end{array}$ & I 2.8 & 10.5 & Br. & 15.8 & $\mathrm{Br}$. & - & $5 \cdot 3$ \\
\hline 1906 I $2 n$ & 3129 & 87.1908 Sagittarii & 651 & -1237.6 & - & Ft. & I 5.2 & 16.0 & $\mathrm{Br}$. & 一 & 0.8 \\
\hline $190810_{n}$ & 3130 & 88.1908 Aquilae & $75^{8}$ & -1035.2 & - & - & I 4.5 & $<15.5$ & - & - & $>1.0$ \\
\hline
\end{tabular}

A list of 22 stars which seem to be certainly variable, but for which there are at present too few plates for confirmation, is given in Table II. The form is nearly the same as that of Table I, except that the designation and Harvard number are omitted.

Table II. Suspected variables.

\begin{tabular}{|c|c|c|c|c|c|c|c|c|c|}
\hline $\begin{array}{l}\text { Prov. Designation } \\
\text { in A. N. }\end{array}$ & RA. 1900 & Decl. I900 & A 2845 & B 23759 & A 4091 & A 5712 & B 32278 & B 3234I & Range \\
\hline 89.I908 Scuti & $18^{\mathrm{h}} 44^{\mathrm{m}} 44^{\mathrm{s}}$ & $-14^{\circ} 4^{2}: 7$ & I 5.0 & - & 15.7 & Br. & Br. & Br. & 0.7 \\
\hline 90.1908 Scuti & $1845 \quad 45$ & $\begin{array}{lll}-12 & 30.5\end{array}$ & Ft. & Ft. & 15.2 & $<$ I 5.9 & Br. & - & $>0.7$ \\
\hline 9 I.1908 Scuti & 1847 I 4 & $\begin{array}{ll}-12 & 43.3\end{array}$ & Ft. & Ft. & $15 \cdot 3$ & I 5.8 & Br. & Ft. & 0.5 \\
\hline $92 . x 908$ Scuti & $18474 \mathrm{I}$ & - II $57 \cdot 3$ & $\mathrm{Br}$. & - & 15.5 & 15.9 & Br. & - & 0.4 \\
\hline 93.1908 Scuti & $1848 \quad 48$ & -1358.9 & Br. & - & 15.2 & 15.8 & - & $\mathrm{Br}$. & 0.6 \\
\hline 94.1908 Scuti & $1849 \quad 47$ & -1254.7 & 15.0 & Ft. & 14.3 & Ft. & Ft. & - & 0.7 \\
\hline 95.1908 Scuti & 1850 I 6 & -1321.1 & 5.3 & Ft. & 14.8 & Ft. & $\mathbf{F t}$. & - & 0.5 \\
\hline 96.1908 Scuti & $\begin{array}{lll}18 & 51 & 2\end{array}$ & $-104 \mathrm{r} .5$ & I 3.7 & $\mathrm{Br}$. & - & 14.5 & Ft. & $\mathbf{F t}$. & 0.8 \\
\hline 97.1908 Scuti & 185120 & -1447.7 & $\mathrm{Br}$. & $\mathrm{Br}$. & 15.7 & 15.2 & $\mathrm{Br}$. & Br. & 0.5 \\
\hline 98.1908 Scuti & $18 \quad 527$ & $\begin{array}{ll}-13 & 2.8\end{array}$ & 14.2 & Ft. & Ft. & I 5.5 & Ft. & Ft. & 1.3 \\
\hline 99.1908 Aquilae & $1853 \quad 58$ & $\begin{array}{ll}-1143 \cdot 3 \\
\end{array}$ & Ft. & Ft. & 15.2 & 16.0 & Ft. & - & 0.8 \\
\hline 100.1908 Sagittarii & 185533 & - I4 6.6 & $\mathrm{Br}$. & - & 15.2 & $<16.0$ & - & - & $>0.8$ \\
\hline I0I.r 908 Sagittarii & $1855 \quad 55$ & -1433.0 & 15.2 & Br. & Br. & 15.8 & Br. & - & 0.6 \\
\hline 102.1908 Sagittarii & $18 \quad 5557$ & $\begin{array}{ll}-14 & 7 \cdot 4\end{array}$ & Ft. & - & 13.6 & 14.0 & 一 & - & 0.4 \\
\hline 103.1908 Sagittarii & $\begin{array}{lll} & 56 & 18\end{array}$ & -13 & $<\mathbf{I} 5.0$ & Ft. & $15 \cdot 5$ & 14.0 & Ft. & - & I. 5 \\
\hline 104.1908 Sagittarii & $18 \quad 5810$ & $\begin{array}{ll}-13 & 50.4\end{array}$ & Ft. & Ft. & I 5.0 & 15.6 & - & Ft. & 0.6 \\
\hline 105.1 908 Sagittarii & I 8594 & -148.5 & - & Ft. & 15.5 & 15.0 & Br. & - & 0.5 \\
\hline 106.1908 Sagittarii & $1859 \quad 5$ & -15 & $14 \cdot 3$ & Ft. & Ft. & 13.0 & Ft. & Ft. & 1.3 \\
\hline 107.1908 Sagittarii & $1 8 \longdiv { 5 9 } 2 1$ & $-I_{2}$ & Ft. & Ft. & r 4.8 & $<15.8$ & Ft. & Ft. & $>1.0$ \\
\hline 108.1908 Sagittarii & 19059 & $-12 \quad 29.5$ & Ft. & Ft. & $<16.0$ & 16.0 & $\mathbf{F t}$. & 14.0 & $>2.0$ \\
\hline I09.1908 Sagittarii & 354 & - I 437.6 & - & Ft. & 15.8 & r 4.8 & Ft. & Ft. & I.0 \\
\hline 1 10.1908 Sagittarii & 537 & - I3 40.4 & - & - & r 5.8 & I 5.0 & - & - & 0.8 \\
\hline
\end{tabular}

Remarks.

$18^{\mathrm{h}} 44^{\mathrm{m}} 44^{\mathrm{s}}$ This star is certainly fainter on A $409 \mathrm{r}$ than on the other plates.

$18^{\mathrm{h}} 45^{\mathrm{m}} 45^{\mathrm{s}}$ A fifteenth magnitude star follows about 0.5 .

$18^{\mathrm{h}} 59^{\mathrm{m}} 21^{\mathrm{s}}$ A sixteenth magnitude star is about $3^{\prime \prime}$ south.

The number of variables contained in the two tables is $\mathbf{r} \mathrm{r}$, and it should be observed that it is undoubtedly only the small number of plates available which prevents the majority of the objects in Table II from being confirmed as certainly variable. In brightness and range, these variables are comparable with those in the Magellanic Clouds. The large number discovered causes the region to be of special interest. Among other regions rich in faint stars may be mentioned those surrounding the Nebula in Carina, the Trifid Nebula, and the Great Spiral Nebula in Cygnus, whose most conspicuous portions are known as NGC. 6960 and $19^{\mathrm{h}} \mathrm{o}^{\mathrm{m}} 59^{\mathrm{s}}$ This star was noticed as bright on $\mathrm{B} 3234 \mathrm{I}$, while observing the variable I $901{ }^{2} \mathrm{~b}_{n}$, given in Table $I$. It seems to show slight fluctuations on other plates.
NGC. 6992. Each of these regions has been examined on a larger number of plates of excellent quality than were available for the locality under discussion, which results given in Circulars 79, 9r, 107, and 115 (A. N. 166.35, I67. 65, I70.3 I7, I7I.343), respectively. Sixteen variables were found in Cygnus and sixteen near the Trifid Nebula, while thirty-two were found in Carina on six plates, all of which have the same centre. The region surrounding Nova Sagittarii appears to exceed all of these in its richness in variable stars. 